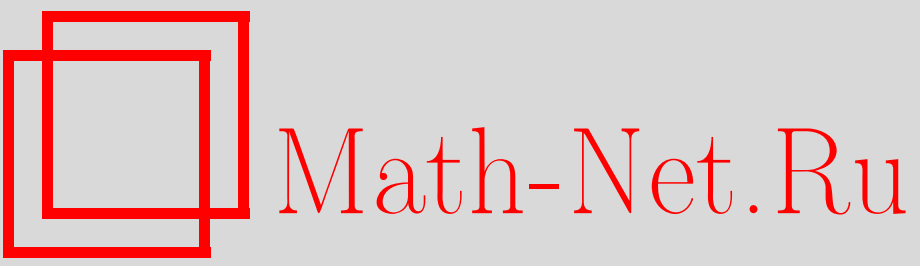

Б. М. Зупник, Геометрия решений $N=2$ SYM-теории в гармоническом суперпространстве, ТМФ, 2002, том 130, номер 2, 251-266

DOI: https://doi.org/10.4213/tmf300

Использование Общероссийского математического портала Math-Net.Ru подразумевает, что вы прочитали и согласны с пользовательским соглашением

http://www.mathnet.ru/rus/agreement

Параметры загрузки:

IP: 54.224 .187 .69

26 апреля 2023 г., 02:17:27 
ТЕОРЕТИЧЕСКАЯ

И МАТЕМАТИЧЕСКАЯ

ФИЗИКА

Том 130, № 2

февраль, 2002

(C) 2002 г

Б. М. Зупник*

\section{ГЕОМЕТРИЯ РЕШЕНИЙ $N=2$ SYМ-ТЕОРИИ В ГАРМОНИЧЕСКОМ СУПЕРПРОСТРАНСТВЕ}

В гармоническом суперпространстве анализируются классические уравнения движения $D=4, \quad N=2$ суперсимметричной теории Янга-Миллса (SYM) для пространств Минковского и Евклида. Изучаются дуальные суперполевые представления уравнений и дополнительных условий, соответствующих классическим SYM-решениям с различными симметриями. В частности, в рамках гармонического подхода описаны альтернативные суперполевые конструкции самодуальных и статических решений.

\section{1. ВВЕДЕНИЕ}

Внемассовые суперполевые связи $N=2$ суперсимметричной теории Янга-Миллса (SYM) в $D=(3,1)$ пространстве Минковского были решены в рамках гармонического суперпространства (HSS) с использованием вспомогательных координат однородного пространства $S_{2} \sim S U_{A}(2) / U_{A}(1)$, где $S U_{A}(2)$ - соответствуюшая группа автоморфизмов [1]-[3]. Аналогичный гармонический формализм можно применить для описания внемассовых $N=2$ суперсимметричных моделей в евклидовом $D=4$ пространстве. Мы будем использовать обозначения с индексами: $\mathrm{HSS}_{\mathrm{M}}$ или $\mathrm{HSS}_{\mathrm{E}}$ для структур HSS над пространствами Минковского или Евклида, соответственно. Вспомогательные гармонические координаты используются в ковариантных условиях грассмановой (G) аналитичности. Неограниченные суперполя $N=2$ теорий определены в $\mathrm{G}$-аналитических суперпространствах с четырьмя грассмановыми координатами.

Гармонические переменные, связанные с пространством $S U_{R}(2) / U_{R}(1)$ для подгруппы евклидовой группы врашений, использовались для изучения автодуальных решений YM- и SYM-теорий [4]-[6]. Эти HSS имеют редуцированные значения четных и нечетных размерностей, а соответствующие аналитические суперполя параметризуют пространства модулей автодуальных решений.

Суперполевые уравнения движения в наиболее общем гармоническом формализме можно эффективно использовать для анализа геометрии классических $N=2$ SYM-решений. В частности, можно изучать дуальные преобразования $N=2 \mathrm{SYM-суперполе-}$ вых переменных в HSS, которые позволяют формулировать необычные представления

* Лаборатория теоретической физики им. Н.Н. Боголюбова, Объединенный институт ядерных исследований, Дубна, Московская обл., Россия. E-mail: zupnik@thsun1.jinr.ru 
уравнений движения и простые калибровочные условия [7], [8]. Важно подчеркнуть, что часть суперполевых связей и уравнений движения в гармоническом SYM-формализме в результате дуальной замены переменных преобразуются в линейные ограничения на HSS-связность $V^{--}$, тогда уравнение нулевой кривизны для гармонических связностей можно интерпретировать как динамическое уравнение. Дуальные представления HSS-суперполевых переменных позволяют исследовать свойства симметрии различных классов частных решений.

Интересно сравнить альтернативные HSS-описания 4-мерных (4D) автодуальных SYM-решений или решений размерно-редуцированных $D<4$ SYM-уравнений. Мы ана-

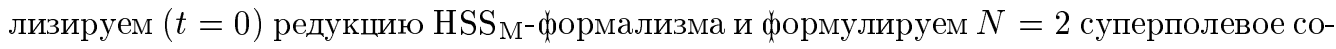
отношение, обобщающее 3D компонентные соотношения Богомольного-Прасада-Зоммерфельда (BPS) для монопольных решений. Показано, что симметрия этих статических суперполевых BPS-соотношений эквивалентна соответствуюшей трехмерной подгруппе евклидовой $N=2$ суперсимметрии. Отметим, что аналогичные суперполевые BPS-соотношения могут быть построены в других вариантах размерно-редуцированных суперсимметричных калибровочных теорий с восемью суперзарядами [9].

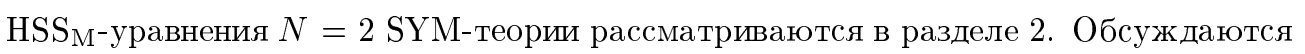
внемассовый формализм с независимыми гармоническими связностями и дуально-эквивалентные конструкции суперполевых уравнений движения. Анализируются условия G-аналитичности для связностей в этом формализме. Суперполевые уравнения движения могут быть преобразованы в соотношения для G-аналитических функций, возникающих в разложениях неаналитической связности $V^{--}$.

Раздел 3 посвящен обсуждению статической 3D редукции $N=2$ суперполевых SYM-уравнений. Временна́я компонента $4 \mathrm{D}$ суперполевой связности $A_{t}$ становится новым 3D скалярным суперполем в этом пределе. Мы рассматриваем 3D суперполевое BPS-соотношение между $A_{t}$ и суперполевыми напряженностями $W$ и $\bar{W}$, которое эквивалентно дифференциальной связи второго порядка для $V^{--}$. Показано, что эта связь второго порядка порождает стандартную связь четвертого порядка для той же связности, и все решения суперполевого BPS-соотношения удовлетворяют SYM-уравнению.

В разделе 4 мы изучаем 4D евклидову версию гармонического формализма. Киральное суперполевое условие автодуальности в этом подходе можно интерпретировать как связь второго порядка для $V^{--}$. Альтернативное представление связностей через суперполевую матрицу перехода (мост-матрицу) и нильпотентное калибровочное условие для этой матришы используются при анализе автодуальных решений в $\mathrm{HSS}_{\mathrm{E}}$.

Сравнение этих гармонических конструкций с аналогичными автодуальными $N=2$ решениями в альтернативном $S U_{R}(2) / U_{R}(1)$-формализме проводится в разделе 5 . Этот вариант гармонического подхода преобразует условие суперавтодуальности в специфические условия грассманово-бозонной аналитичности гармонической связности на массовой поверхности. Мы рассматриваем полезную идентификацию гармонических переменных для различных подгрупп группы $S U(2)$, которая позволяет связать автодуальные гармонические суперполя в различных подходах. Отметим, что для калибровочной группы $S U(2)$ широкий класс автодуальных HSS-решений можно построить в квадра- 
турах [10].

Статические автодуальные решения изучаются в разделе 6 . Мы рассматриваем связь меж ду сопряженными спинорными координатами в статическом пределе $4 \mathrm{D} \mathrm{HSS}_{\mathrm{M}}$ и соответствуюшими псевдовешественными спинорными координатами в $3 \mathrm{D}$ редукции $\mathrm{HSS}_{\mathrm{E}}$.

Основные формулы, используемые в методе HSS, приведены в приложениях А и Б. Мы описываем правила сопряжения для различных вариантов HSS, которые очень важны для SYM-теории.

\section{2. ПРЕДСТАВЛЕНИЯ $N=2$ SҮМ-УРАВНЕНИЙ В ГАРМОНИЧЕСКОМ СУПЕРПРОСТРАНСТВЕ}

Гармоническое $S U_{A}(2) / U_{A}(1)$-преобразование впервые было использовано для решения внемассовых связей для суперполевых связностей $A_{a}^{k}(z)$ в $N=2, \quad D=(3,1)$ SYM-теории [1], [2]:

$$
u_{k}^{+}\left(D_{a}^{k}+A_{a}^{k}\right) \equiv \nabla_{a}^{+}=e^{-v} D_{a}^{+} e^{v},
$$

где $a=(\alpha, \dot{\alpha})$ - индексы группы $S L(2, \mathbb{C}), \quad k-2$-компонентный индекс группы автоморфизмов $S U_{A}(2), u_{k}^{+}$- вспомогательные гармонические переменные (см. приложение A), $v(z, u)$ - суперполевая мост-матрица. Это преобразование формально связывает представления калибровочной группы в центральном и аналитическом базисах.

Основным внемассовым гармоническим суперполем SYM-теории является связность $V^{++}=e^{v} D^{++} e^{-v}$ для гармонической ковариантной производной в аналитическом базисе

$$
\nabla^{++}=D^{++}+V^{++}, \quad \delta V^{++}=-D^{++} \lambda-\left[V^{++}, \lambda\right] .
$$

Препотенциал $V^{++}$и калибровочные параметры в аналитическом базисе удовлетворяют условиям G-аналитичности

$$
\left(D_{\alpha}^{+}, \bar{D}_{\dot{\alpha}}^{+}\right)\left(V^{++}, \lambda\right)=0 .
$$

G-аналитические суперполя описываются неограниченными функциями аналитических координат $\zeta$ (см. ниже (А.5)) и гармоник $u_{i}^{ \pm}$. Вторая гармоническая связность $V^{--}$ удовлетворяет гармоническому условию нулевой кривизны

$$
D^{++} V^{--}-D^{--} V^{++}+\left[V^{++}, V^{--}\right]=0,
$$

которое имеет точное решение в виде ряда по степеням $V^{++}[3]$.

Спинорные и векторные связности $A_{a}^{ \pm}, A_{\alpha \dot{\beta}}$, а также суперполевые напряженности $W$ и $\bar{W}$ в аналитическом базисе могут быть выражены через спинорные производные связности $V^{--}$:

$$
\begin{gathered}
A_{\alpha}^{+}=\bar{A}_{\dot{\alpha}}^{+}=0, \quad A_{\alpha}^{-}=-D_{\alpha}^{+} V^{--}, \quad \bar{A}_{\dot{\alpha}}^{-}=-\bar{D}_{\dot{\alpha}}^{+} V^{--}, \\
A_{\alpha \dot{\beta}} \equiv i D_{\alpha}^{+} \bar{A}_{\dot{\beta}}^{-} \equiv-i \bar{D}_{\dot{\beta}}^{+} A_{\alpha}^{-}=-i D_{\alpha}^{+} \bar{D}_{\dot{\beta}}^{+} V^{--} \\
\bar{W}=\frac{1}{2} D^{\alpha+} A_{\alpha}^{-}=-\left(D^{+}\right)^{2} V^{--}, \quad W=-\frac{1}{2} \bar{D}_{\dot{\alpha}}^{+} \bar{A}^{\dot{\alpha}-}=\left(\bar{D}^{+}\right)^{2} V^{--} .
\end{gathered}
$$


Нелинейное уравнение движения для препотенциала $V^{++}$может быть сформулировано как линейное дифференциальное условие на композитную связность $V^{--}\left(V^{++}\right)$:

$$
\left(D^{+}\right)^{2}\left(\bar{D}^{+}\right)^{2} V^{--}=0
$$

Уравнение (2.4) трактуется как кинематическое интегрируемое уравнение в стандартном SYM-формализме с независимым суперполем $V^{++}$. Альтернативный $\mathrm{HSS}_{\mathrm{M}}$-формализм с независимым внемассовым суперполем $V^{--}$рассматривался в работах [7]. Этот подход аналогичен формализму первого порядка в обычной теории Янга-Миллса, так как независимое суперполе $V^{--}$вне массовой поверхности содержит бесконечное число вспомогательных полей, включая поля размерности $-2: F, F_{m n}$ и $F_{m}$. (Отметим, что замену независимых HSS-переменных $V^{++} \rightarrow V^{--}$или $V^{++} \rightarrow v$ [8] можно интерпретировать как дуальное преобразование в SYM-теории. Мы надеемся, что богатая структура дуальных преобразований в HSS будет полезна для построения различных классических решений.)

Условие $\mathrm{G}$-аналитичности $V^{++}$эквивалентно нелинейному уравнению аналитичности для связности $V^{--}$

$$
\left[\nabla_{a}^{-}, \nabla^{--}\right]=D_{a}^{-} V^{--}+D^{--} D_{a}^{+} V^{--}+\left[V^{--}, D_{a}^{+} V^{--}\right]=0 .
$$

Линейную связь (2.6) для независимого суперполя $V^{--}$можно легко решить [7]:

$$
V^{--}=D_{\alpha}^{+} B^{\alpha(-3)}-\bar{D}_{\dot{\alpha}}^{+} \bar{B}^{\dot{\alpha}(-3)}
$$

В представлении с независимыми SYM-суперполевыми переменными $B^{\alpha(-3)}$ и $V^{++}$ основное динамическое уравнение имеет вид

$$
\nabla^{++}\left(D_{\alpha}^{+} B^{\alpha(-3)}-\bar{D}_{\dot{\alpha}}^{+} \bar{B}^{\dot{\alpha}(-3)}\right)=D^{--} V^{++} .
$$

Действуя операторами $D_{\alpha}^{+}, \bar{D}_{\dot{\alpha}}^{+}$на обе части этого уравнения, можно получить следуюшие соотношения:

$$
\begin{aligned}
& \nabla^{++}\left(\bar{D}^{+}\right)^{2} D_{\alpha}^{+} B^{\alpha(-3)}=0 \\
& \nabla^{++}\left[\left(\bar{D}^{+}\right)^{2} D_{\alpha}^{+} \bar{B}_{\dot{\alpha}}^{(-3)}-\left(D^{+}\right)^{2} \bar{D}_{\dot{\alpha}}^{+} B_{\alpha}^{(-3)}\right]=i \partial_{\alpha \dot{\alpha}} V^{++}
\end{aligned}
$$

Разложение уравнения (2.9) по всем спинорным координатам дает компонентные SYM-уравнения, однако полезно также исследовать частичное разложение этого суперполевого уравнения по $\theta^{\alpha-}$ и $\bar{\theta}^{\dot{\alpha}-}$. Определим $\mathrm{G}$-аналитические компоненты суперполя $V^{--}$:

$$
\begin{gathered}
\bar{\phi}=-\left(D^{+}\right)^{2} V^{--}\left|, \quad \phi=\left(\bar{D}^{+}\right)^{2} V^{--}\right|, \quad B_{\alpha \dot{\beta}}=\bar{D}_{\dot{\beta}}^{+} D_{\alpha}^{+} V^{--} \mid, \\
\lambda_{\alpha}^{+}=-D_{\alpha}^{+}\left(\bar{D}^{+}\right)^{2} V^{--}\left|, \quad \bar{\lambda}_{\dot{\alpha}}^{+}=-\bar{D}_{\dot{\alpha}}^{+}\left(D^{+}\right)^{2} V^{--}\right|, \\
F^{++}=\left(D^{+}\right)^{2}\left(\bar{D}^{+}\right)^{2} V^{--} \mid
\end{gathered}
$$


где символ $\mid$ означает $\theta^{-}=\bar{\theta}^{-}=0$.

Полное преобразование суперсимметрии $\delta_{\epsilon} V^{--}=0$ порождает соответствуюшие преобразования G-аналитических компонент

$$
\begin{aligned}
\delta_{\epsilon} \phi & =u_{k}^{-} \epsilon^{\alpha k} \lambda_{\alpha}^{+}, & & \delta_{\epsilon} B_{\alpha \dot{\beta}}=u_{k}^{-} \epsilon_{\alpha}^{k} \bar{\lambda}_{\dot{\beta}}^{+}+u_{k}^{-} \bar{\epsilon}_{\dot{\beta}}^{k} \lambda_{\alpha}^{+}, \\
\delta_{\epsilon} \lambda_{\alpha} & =-u_{k}^{-} \epsilon_{\alpha}^{k} F^{++}, & & \delta_{\epsilon} F^{++}=0 .
\end{aligned}
$$

Используя калибровочное преобразование $\delta V^{--}=-D^{--} \lambda-\left[V^{--}, \lambda\right]$, можно получить нильпотентное калибровочное условие для гармонической связности:

$$
\begin{aligned}
\mathcal{V}^{--}= & \left(\theta^{-}\right)^{2} \bar{\phi}-\left(\bar{\theta}^{-}\right)^{2} \phi+\theta^{\alpha-} \bar{\theta}^{\dot{\beta}-} B_{\alpha \dot{\beta}}+\left(\theta^{-}\right)^{2} \bar{\theta}^{\dot{\alpha}-} \bar{\lambda}_{\dot{\alpha}}^{+}+ \\
& +\left(\bar{\theta}^{-}\right)^{2} \theta^{\alpha-} \lambda_{\alpha}^{+}+\left(\theta^{-}\right)^{2}\left(\bar{\theta}^{-}\right)^{2} F^{++} .
\end{aligned}
$$

Геометрические суперполя в этой калибровке имеют вид

$$
\begin{aligned}
W & =\phi-\theta^{\alpha-} \lambda_{\alpha}^{+}-\left(\theta^{-}\right)^{2} F^{++} \\
\bar{W} & =\bar{\phi}+\bar{\theta}^{\dot{\alpha}-} \bar{\lambda}_{\dot{\alpha}}^{+}+\left(\bar{\theta}^{-}\right)^{2} F^{++} \\
A_{\alpha \dot{\beta}} & =-i B_{\alpha \dot{\beta}}+i \theta_{\alpha}^{-} \bar{\lambda}_{\dot{\beta}}^{+}+i \bar{\theta}_{\dot{\beta}}^{-} \lambda_{\alpha}^{+}-i \theta_{\alpha}^{-} \bar{\theta}_{\dot{\beta}}^{-} F^{++} .
\end{aligned}
$$

Связь $F^{++}=0$ дает следуюшие аналитические уравнения движения, эквивалентные уравнению (2.9):

$$
\begin{gathered}
\nabla^{++} \phi=\theta^{\alpha+} \lambda_{\alpha}^{+}, \quad\left(\nabla^{++}\right)^{2} \phi=0, \\
\nabla^{++} B_{\alpha \dot{\beta}}=-i \partial_{\alpha \dot{\beta}} \mathcal{V}^{++}+\theta_{\alpha}^{+} \bar{\lambda}_{\dot{\beta}}^{+}+\bar{\theta}_{\dot{\beta}}^{+} \lambda_{\alpha}^{+}, \\
\nabla^{++} \lambda_{\alpha}^{+}=0 .
\end{gathered}
$$

Компонентные решения для аналитических $N=2$ суперполей на массовой поверхности имеют вид

$$
\begin{aligned}
\phi= & \varphi(x)+\theta^{\alpha+} u_{k}^{-} \psi_{\alpha}^{k}(x), \\
B_{\alpha \dot{\beta}}= & a_{\alpha \dot{\beta}}(x)+\theta_{\alpha}^{+} u_{k}^{-} \bar{\psi}_{\dot{\beta}}^{k}(x)+\bar{\theta}_{\dot{\beta}}^{+} u_{k}^{-} \psi_{\alpha}^{k}(x), \\
\mathcal{V}^{++}= & \left(\theta^{+}\right)^{2} \bar{\varphi}(x)-\left(\bar{\theta}^{+}\right)^{2} \varphi(x)+\theta^{\alpha+} \bar{\theta}^{\dot{\beta}+} a_{\alpha \dot{\beta}}(x)- \\
& -\theta^{\alpha+}\left(\bar{\theta}^{+}\right)^{2} u_{k}^{-} \psi_{\alpha}^{k}(x)-\bar{\theta}^{\dot{\alpha}+}\left(\theta^{+}\right)^{2} u_{k}^{-} \bar{\psi}_{\dot{\alpha}}^{k}(x) .
\end{aligned}
$$

$N=2$ уравнения движения для этих компонент следуют из G-аналитических суперполевых уравнений. Разумеется, главная задача гармонического подхода - найти нестандартные суперполевые методы решения SYM-уравнений, которые не могут быть полностью сведены к анализу компонентных уравнений. Мы надеемся, что сопоставление дуальных суперполевых представлений SYM-решений будет полезно для поиска необычных свойств симметрии, которые не видны непосредственно в компонентном представлении. 


\section{3. СТАТИЧЕСКИЕ СУПЕРПОЛЕВЫЕ УРАВНЕНИЯ}

Трехмерные монопольные решения играют важную роль в современных непертурбативных методах квантовой $N=2$ SYM-теории [11], [12]. Гармонический анализ монопольных решений в теории Янга-Миллса рассматривался в работе [5]. Мы будем изучать альтернативные HSS-конструкции статических $N=2 \mathrm{SYM-решений} \mathrm{и} \mathrm{их} \mathrm{связь} \mathrm{с}$ 4D автодуальными решениями.

Рассмотрим нерелятивистское представление координат $D=(3,1), N=2$ HSS, основанное на статической групше $S O(3)$ :

$$
\begin{aligned}
x_{A}^{\alpha \dot{\beta}} & \rightarrow i y_{\beta}^{\alpha}+\delta_{\beta}^{\alpha} t_{A}, \quad y_{\alpha}^{\alpha}=0, \quad y^{\alpha \beta}=\varepsilon^{\alpha \rho} y_{\rho}^{\beta}, \\
\partial_{\alpha \dot{\beta}} & \rightarrow-i \partial_{\beta}^{\alpha}+\delta_{\alpha}^{\beta} \partial_{t}, \quad \partial_{\gamma}^{\sigma} y_{\beta}^{\alpha}=2 \delta_{\beta}^{\sigma} \delta_{\gamma}^{\alpha}-\delta_{\beta}^{\alpha} \delta_{\gamma}^{\sigma}, \\
\bar{\theta}^{\dot{\alpha} \pm} & \rightarrow \bar{\theta}_{\alpha}^{ \pm}, \quad \bar{\theta}^{\alpha \pm}=\varepsilon^{\alpha \beta} \bar{\theta}_{\beta}^{ \pm}, \\
\bar{\partial}_{\dot{\alpha}}^{ \pm} & \rightarrow \bar{\partial}^{\alpha \pm}, \quad \bar{\partial}^{\alpha \pm} \bar{\theta}_{\beta}^{\mp}=\delta_{\beta}^{\alpha} .
\end{aligned}
$$

Мы будем использовать следуюшие правила сопряжения:

$$
\begin{aligned}
\left(y_{\beta}^{\alpha}\right)^{\dagger} & =-y_{\alpha}^{\beta}, & & \left(y^{\alpha \beta}\right)^{\dagger} & =y_{\alpha \beta}, & \left(t_{A}\right)^{\dagger}=t_{A}, \\
\left(\theta^{\alpha \pm}\right)^{\dagger} & =\bar{\theta}_{\alpha}^{ \pm}, & \left(\theta_{\alpha}^{ \pm}\right)^{\dagger} & =-\bar{\theta}^{\alpha \pm}, & & \left(\varepsilon^{\alpha \beta}\right)^{\dagger}=-\varepsilon_{\alpha \beta}, \\
\left(\bar{\theta}^{\alpha \pm}\right)^{\dagger} & =\theta_{\alpha}^{ \pm}, & \left(\bar{\theta}_{\alpha}^{ \pm}\right)^{\dagger} & =-\theta^{\alpha \pm}, & & \\
\left(D_{\alpha}^{ \pm}\right)^{\dagger} & =\bar{D}^{\alpha \pm}, & & \left(\bar{D}^{\alpha \pm}\right)^{\dagger}=-D_{\alpha}^{ \pm}, & & \left(\bar{D}_{\alpha}^{ \pm}\right)^{\dagger}=D^{\alpha \pm} .
\end{aligned}
$$

Отметим, что изменение положения точечных $S L(2, \mathbb{C})$-индексов после статической редукции связано с соглашением о сопряжении $S U(2)$-спиноров, которое преврашает верхние индексы в нижние.

Редукция времени $(t=0)$ преврашает 4D гармонические суперполя в $3 \mathrm{D}$ евклидовы суперполя, ковариантные относительно редуцированной суперсимметрии с восемью суперзарядами.

Рассмотрим соответствуюшее представление 4-векторной связности

$$
A_{\alpha \dot{\beta}} \Rightarrow A_{\alpha}^{\beta}+\delta_{\alpha}^{\beta} A_{t}, \quad\left(A_{\alpha}^{\beta}\right)^{\dagger}=-A_{\beta}^{\alpha}, \quad\left(A_{t}\right)^{\dagger}=-A_{t},
$$

где временна́я компонента связности становится ковариантной по отношению к остаточным статическим калибровочным преобразованиям

$$
\begin{aligned}
& A_{\alpha}^{\beta}=i\left(\bar{D}^{\beta+} D_{\alpha}^{+}-\frac{1}{2} \delta_{\alpha}^{\beta}\left(\bar{D}^{+} D^{+}\right)\right) V^{--}, \\
& A_{t}=\frac{i}{2}\left(\bar{D}^{+} D^{+}\right) V^{--}, \quad \delta_{\lambda} A_{t}=\left[\lambda, A_{t}\right] .
\end{aligned}
$$

Для анализа свойств симметрии суперсимметричных монопольных решений представляется полезным построение явно суперсимметричного обобшения BPS-уравнения 
для $N=2$ калибровочной теории. В дополнение к $t=0$ редукции HSS-уравнения (2.4) мы предлагаем рассмотреть следуюшее суперполевое BPS-соотношение:

$$
S \equiv-2 \operatorname{ir} A_{t}+p(W+\bar{W})=0,
$$

где $r$ и $p$ - некоторые вешественные параметры. Отметим, что это соотношение нарушает $R$-симметрию действия $\theta^{ \pm} \rightarrow e^{i \rho / 2} \theta^{ \pm}, W \rightarrow e^{i \rho} W$.

Будем трактовать соотношение (3.5) как линейную связь второго порядка на $V^{--}$

$$
S=\left[r\left(\bar{D}^{+} D^{+}\right)+p\left(\bar{D}^{+}\right)^{2}-p\left(D^{+}\right)^{2}\right] V^{--}=0,
$$

которая также дает спинорное условие третьего порядка

$$
\left(-r\left(D^{+}\right)^{2} \bar{D}_{\alpha}^{+}+p\left(\bar{D}^{+}\right)^{2} D_{\alpha}^{+}\right) V^{--}=0 .
$$

Для случая $r \neq p$ последнее уравнение для спиноров не будет вырожденным, поэтому можно получить сильные ограничения:

$$
D_{\alpha}^{+}\left(\bar{D}^{+}\right)^{2} V^{--}=D_{\alpha}^{+} W=0 \Rightarrow \nabla^{--} D_{\alpha}^{+} W=\nabla_{\alpha}^{-} W=0 .
$$

Очевидно, что соответствуюшее ковариантно-постоянное решение $W=$ const coхраняет все восемь суперзарядов [13], однако для этого тривиального решения с постоянными скалярными полями все фермионы и калибровочные поля равны нулю. Вырожденный случай $r=p$ соответствует условию самосопряженности для $3 \mathrm{D}$ спиноров

$$
\lambda_{k}^{\alpha}=\bar{\lambda}_{k}^{\alpha}=\varepsilon^{\alpha \beta} \varepsilon_{k l}\left(\lambda_{l}^{\beta}\right)^{\dagger} .
$$

Соответствуюшие автодуальные статические решения будут обсуждаться в разделе 6 .

\section{4. ЕВКЛИДОВЫ $N=2$ SУМ-УРАВНЕНИЯ}

Мы будем анализировать суперполевые связи $N=2$ SYM-теории в евклидовом суперпространстве

$$
\begin{aligned}
\left\{\nabla_{\alpha}^{k}, \nabla_{\beta}^{l}\right\} & =\varepsilon_{\alpha \beta} \varepsilon^{k l} \mathcal{W}, \\
\left\{\widehat{\nabla}_{k \dot{\alpha}}, \widehat{\nabla}_{l \dot{\beta}}\right\} & =\varepsilon_{\dot{\alpha} \dot{\beta}} \varepsilon_{k l} \widehat{\mathcal{W}}, \\
\left\{\nabla_{\alpha}^{k}, \widehat{\nabla}_{l \dot{\beta}}\right\} & =\delta_{l}^{k} \nabla_{\alpha \dot{\beta}},
\end{aligned}
$$

где $\mathcal{W}$ и $\widehat{\mathcal{W}}$ - независимые вещественные суперполевые напряженности, а пары индексов $\alpha, \beta ; \dot{\alpha}, \dot{\beta}$ и $k, l$ описьвают соответственно 2-спинорные представления евклидовой группы $S U_{L}(2) \times S U_{R}(2)$ и группы автоморфизмов $S U_{A}(2)$.

Суперполевые аналоги евклидовых уравнений автодуальности рассматривались в работах [14]-[17]. Мы будем анализировать $N=2$ автодуальные решения в рамках

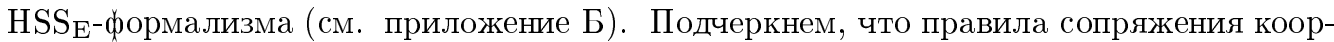
динат и производных $N=2$ суперпространства сушественно различаются для случаев евклидовой группы $S O(4)$ и группы Лоренца $S O(3,1)$. 
Описание евклидовой $N=2$ SYM-теории в $S U_{A}(2) / U_{A}(1)$ HSS похоже на соответствуюший формализм в $\mathrm{HSS}_{\mathrm{M}}$, в частности можно использовать те же обозначения $V^{++}$, $V^{--}$для евклидовых гармонических связностей. Евклидовы спинорные и векторные связности аналитического базиса имеют следующий вид:

$$
\begin{gathered}
\mathcal{A}_{\alpha}^{+}=\hat{\mathcal{A}}_{\dot{\alpha}}^{+}=0, \quad \mathcal{A}_{\alpha}^{-}=-\mathcal{D}_{\alpha}^{+} V^{--}, \quad \hat{\mathcal{A}}_{\dot{\alpha}}^{-}=-\widehat{\mathcal{D}}_{\dot{\alpha}}^{+} V^{--}, \\
\mathcal{B}_{\alpha \dot{\beta}} \equiv \mathcal{D}_{\alpha}^{+} \hat{\mathcal{A}}_{\dot{\beta}}^{-} \equiv \mathcal{D}_{\alpha}^{+} \widehat{\mathcal{D}}_{\dot{\beta}}^{+} V^{--}
\end{gathered}
$$

Вещественность евклидовых суперполевых связностей очевидна в $\mathrm{HSS}_{\mathrm{E}}$ :

$$
\mathcal{W}=\left(\mathcal{D}^{+}\right)^{2} V^{--}, \quad \widehat{\mathcal{W}}=\left(\widehat{\mathcal{D}}^{+}\right)^{2} V^{--} .
$$

Система уравнений автодуальности в HSS имеет следующий вид:

$$
\mathcal{D}^{++} V^{--}+\left[V^{++}, V^{--}\right]=\mathcal{D}^{--} V^{++}, \quad\left(\mathcal{D}^{+}\right)^{2} V^{--}=0 .
$$

Простое решение линейной связи для $V^{--}$,

$$
V^{--}=\mathcal{D}_{\alpha}^{+} \mathcal{B}^{\alpha(-3)}
$$

преврашает гармоническое уравнение в соотношение между $\mathcal{B}^{\alpha(-3)}$ и $V^{++}$:

$$
\mathcal{D}_{\alpha}^{+} \nabla^{++} \mathcal{B}^{\alpha(-3)}=\mathcal{D}^{--} V^{++} .
$$

Уравнение автодуальности эквивалентно соотношению

$$
\mathcal{D}_{\alpha}^{+} \mathcal{A}_{\beta}^{-}=0 .
$$

Используя уравнение $\left[\nabla^{--}, \nabla_{\alpha}^{-}\right]=0$, можно получить условия киральности

$$
\left\{\nabla_{\alpha}^{ \pm}, \nabla_{\beta}^{ \pm}\right\}=0
$$

Рассмотрим представление гармонических связностей через мост-матрицу $v[1]$ :

$$
V^{ \pm \pm} \equiv e^{v} \mathcal{D}^{ \pm \pm} e^{-v}
$$

Условие автодуальности второго порядка для связности $\left(\mathcal{D}^{+}\right)^{2} V^{--}=0($ см. $(4.4))$ соответствует дифферециальному условию первого порядка для автодуальной матрищы $v$

$$
\mathcal{D}_{\alpha}^{+} v=0,
$$

которое позволяет решить связь (4.7):

$$
\mathcal{A}_{\beta}^{-}=-\mathcal{D}_{\alpha}^{+}\left(e^{v} \mathcal{D}^{--} e^{-v}\right)=e^{v} \mathcal{D}_{\alpha}^{-} e^{-v}, \quad \mathcal{D}_{\alpha}^{+} \mathcal{A}_{\beta}^{-}=0 .
$$


Сравним представления (4.5) и (4.9) и получим соотношение

$$
\mathcal{B}^{\alpha(-3)}=\frac{1}{2} \Theta^{\alpha-} e^{v} \mathcal{D}^{--} e^{-v}+\frac{1}{2}\left(\Theta^{-}\right)^{2} e^{v} \mathcal{D}^{\alpha-} e^{-v}
$$

Гармонические проекции связностей центрального базиса в этом представлении имеют следующий вид:

$$
\begin{aligned}
& A_{\alpha}^{ \pm}=0, \quad \hat{A}_{\dot{\alpha}}^{+}(v)=e^{-v} \widehat{\mathcal{D}}_{\dot{\alpha}}^{+} e^{v} \\
& \hat{A}_{\dot{\alpha}}^{-}=\mathcal{D}^{--} \hat{A}_{\dot{\alpha}}^{+}(v), \quad B_{\alpha \dot{\alpha}}(v)=\mathcal{D}_{\alpha}^{-} \hat{A}_{\dot{\alpha}}^{+}(v) .
\end{aligned}
$$

Это автодуальное представление ковариантно относительно преобразований $e^{v} \Rightarrow$ $e^{\lambda} e^{v} e^{\tau_{r}}$, где $\lambda-\mathrm{G}$-аналитический параметр, а $\tau_{r}(z)-$ киральный параметр, $D_{\alpha}^{i} \tau_{r}=$ $\mathcal{D}^{ \pm \pm} \tau_{r}=0$.

Следует подчеркнуть, что для автодуальных решений суперполе $v$ в общем случае не должно быть киральным, т.е. $\mathcal{D}_{\alpha}^{-} v \neq 0$. Очевидно, что киральные суперполя $v$ соответствуют тривиальным решениям $\mathcal{D}_{\alpha}^{+} \widehat{W}=-\left(\widehat{\mathcal{D}}^{+}\right)^{2} e^{v} \mathcal{D}_{\alpha}^{-} e^{-v}=0$.

В представлении (4.9) система уравнений автодуальности (4.4) дает нелинейное уравнение для $v$

$$
\widehat{\mathcal{D}}_{\dot{\alpha}}^{+}\left(e^{v} D^{++} e^{-v}\right) \equiv \widehat{\mathcal{D}}_{\dot{\alpha}}^{+} V^{++}(v)=0
$$

или эквивалентное уравнение центрального базиса $\mathcal{D}^{++} \hat{A}_{\dot{\alpha}}^{+}(v)=0$. Из этого главного уравнения $\mathrm{G}$-аналитичности мы можем получить следуюшие соотношения:

$$
\begin{aligned}
\mathcal{D}^{++} A_{\alpha \dot{\beta}}(v) & =0, \quad \mathcal{D}_{\alpha}^{+} B_{\beta \dot{\beta}}(v)=0 \\
\mathcal{D}_{\alpha}^{-} B_{\beta \dot{\beta}}(v) & =\varepsilon_{\alpha \beta}\left(\mathcal{D}^{-}\right)^{2}\left[e^{-v} \widehat{\mathcal{D}}_{\dot{\alpha}}^{+} e^{v}\right]=0 .
\end{aligned}
$$

Автодуальная матрица $v$, удовлетворяюшая условию частичной $\mathrm{G}$-аналитичности (4.10), не зависит от грассмановых координат $\Theta^{\alpha-}$. Введем нильпотентное калибровочное условие для этой матрицы:

$$
v=\widehat{\Theta}^{\dot{\alpha}-} b_{\dot{\alpha}}^{+}+\left(\widehat{\Theta}^{-}\right)^{2} b^{++}, \quad v^{2}=-\left(\widehat{\Theta}^{-}\right)^{2} b^{\dot{\alpha}+} b_{\dot{\alpha}}^{+}
$$

где $b_{\dot{\alpha}}^{+}$и $b^{++}-\mathrm{G}$-аналитические матричные коэффициенты. Отметим, что аналогичное нильпотентное представление мост-матрицы было недавно использовано в $N=3$ SYM-теории [18].

Построим гармоническую связность $V^{++}(v)$

$$
\begin{aligned}
e^{v} D^{++} e^{-v}= & -\widehat{\Theta}^{\dot{\alpha}+} b_{\dot{\alpha}}^{+}+\widehat{\Theta}^{\dot{\alpha}-}\left(-D^{++} b_{\dot{\alpha}}^{+}-\widehat{\Theta}_{\dot{\alpha}}^{+} b^{++}+\frac{1}{2} \widehat{\Theta}^{\dot{\beta}+}\left\{b_{\dot{\alpha}}^{+}, b_{\dot{\beta}}^{+}\right\}\right)+ \\
& +\left(\widehat{\Theta}^{-}\right)^{2}\left(D^{++} b^{++}+\frac{1}{2}\left\{b^{\dot{\alpha}+}, D^{++} b_{\dot{\alpha}}^{+}\right\}+\right. \\
& \left.+\widehat{\Theta}^{\dot{\alpha}+}\left[b_{\dot{\alpha}}^{+}, b^{++}\right]+\frac{1}{2} \widehat{\Theta}^{\dot{\alpha}+}\left\{b_{\dot{\alpha}}^{+}, b^{\dot{\beta}+} b_{\dot{\beta}}^{+}\right\}\right)
\end{aligned}
$$


где мы использовали тождество

$$
\left\{b_{\dot{\alpha}}^{+}, b^{\dot{\beta}+} b_{\dot{\beta}}^{+}\right\}=\frac{1}{3}\left[b^{\dot{\beta}+},\left\{b_{\dot{\alpha}}^{+}, b_{\dot{\beta}}^{+}\right\}\right] .
$$

Уравнение динамической аналитичности

$$
V^{++}(v)=-\widehat{\Theta}^{\dot{\alpha}+} b_{\dot{\alpha}}^{+}
$$

порождает следуюшие аналитические соотношения:

$$
\begin{gathered}
D^{++} b_{\dot{\alpha}}^{+}+\widehat{\Theta}_{\dot{\alpha}}^{+} b^{++}-\frac{1}{2} \widehat{\Theta}^{\dot{\beta}+}\left\{b_{\dot{\alpha}}^{+}, b_{\dot{\beta}}^{+}\right\}=0 \\
D^{++} b^{++}+\frac{1}{2}\left\{b^{\dot{\alpha}+}, D^{++} b_{\dot{\alpha}}^{+}\right\}+\widehat{\Theta}^{\dot{\alpha}+}\left[b_{\dot{\alpha}}^{+}, b^{++}\right]+\frac{1}{6} \widehat{\Theta}^{\dot{\alpha}+}\left[b^{\dot{\beta}+},\left\{b_{\dot{\alpha}}^{+}, b_{\dot{\beta}}^{+}\right\}\right]=0 .
\end{gathered}
$$
$\widehat{\Theta}^{\dot{\alpha}+}$

Будем использовать промежуточное разложение $(2,2)$-аналитических суперполей по

$$
\begin{aligned}
b_{\dot{\alpha}}^{+} & =\beta_{\dot{\alpha}}^{+}+\widehat{\Theta}^{\dot{\beta}+} C_{\dot{\alpha} \dot{\beta}}+\left(\widehat{\Theta}^{+}\right)^{2} \eta_{\dot{\alpha}}^{-}, \\
b^{++} & =B^{++}+\widehat{\Theta}^{\dot{\alpha}+} \gamma_{\dot{\alpha}}^{+}+\left(\widehat{\Theta}^{+}\right)^{2} B
\end{aligned}
$$

где все коэффициенты представляют собой полиномы по $\Theta^{\alpha+} .(2,2)$-уравнения для $b_{\dot{\alpha}}^{+}$и $b^{++}$дают $(2,0)$-уравнения для этих коэффициентов, например

$$
\begin{gathered}
\partial^{++} \beta_{\dot{\alpha}}^{+}=0, \quad \partial^{++} B^{++}=0, \\
\partial^{++} C_{\dot{\alpha} \dot{\beta}}+\Theta^{\beta+} \partial_{\beta \dot{\beta}} \beta_{\dot{\alpha}}^{+}+\varepsilon_{\dot{\alpha} \dot{\beta}} B^{++}-\frac{1}{2}\left\{\beta_{\dot{\alpha}}^{+}, \beta_{\dot{\beta}}^{+}\right\}=0 .
\end{gathered}
$$

Линейные гармонические уравнения для $\beta_{\dot{\alpha}}^{+}$и $B^{++}$можно решить явно. Неоднородное линейное гармоническое уравнение для $C_{\dot{\alpha} \dot{\beta}}$ содержит источник, составленный из суперполей, вычисленных на предыдущем этапе, поэтому оно в принципе интегрируемо. Неоднородные гармонические уравнения для других $(2,0)$-коэффициентов $\eta_{\dot{\alpha}}^{-}, \gamma_{\dot{\alpha}}^{+}$и $B$ могут быть получены и решены аналогично. Следует подчеркнуть, что автодуальные уравнения (4.4) и (4.19) удобны для поиска размерно-редуцированных решений.

\section{5. ГАРМОНИЧЕСКИЕ КОНСТРУКЦИИ АВТОДУАЛЬНЫХ РЕШЕНИЙ}

Альтернативный гармонический формализм для автодуальных SYM-решений рассматривался в работе [6]. Этот формализм гармонизует одну из пространственных групп - группу $S U_{R}(2)$, действующую на индексах $\dot{\alpha}$.

Чтобы сравнить альтернативные гармонические подходы к $N=2$ автодуальным уравнениям, рассмотрим нековариантную процедуру идентификации $S U_{R}(2)=S U_{A}(2)$ для решений этих уравнений. Отождествим также соответствующие спинорные индексы $\dot{\alpha} \equiv l$. Координаты $N=2$ суперпространства в этих обозначениях имеют вид

$$
y^{\alpha \dot{\alpha}} \equiv y^{\alpha l}, \quad \widehat{\Theta}^{\dot{\alpha} k} \equiv \widehat{\Theta}^{l, k} .
$$


Используя единственный набор $S U(2) / U(1)$-гармоник $u_{i}^{ \pm}$, можно рассмотреть гармонические проекции центральных $y$, аналитических $Y$ и киральных $y_{r}$ координат, а также грассмановых координат $\widehat{\Theta}$ :

$$
\begin{aligned}
y^{\alpha \pm} & =u_{k}^{ \pm} y^{\alpha k}, \quad Y^{\alpha \pm}=y^{\alpha \pm}-\Theta^{\alpha+} \widehat{\Theta}^{ \pm,-}-\Theta^{\alpha-} \widehat{\Theta}^{ \pm,+}, \\
y_{r}^{\alpha \pm} & =y^{\alpha \pm}+\Theta^{\alpha+} \widehat{\Theta}^{ \pm,-}-\Theta^{\alpha-} \widehat{\Theta}^{ \pm,+} \\
\Theta^{\alpha \pm} & =u^{k \pm} \Theta_{k}^{\alpha}, \quad \widehat{\Theta}^{ \pm, \pm}=u_{l}^{ \pm} u_{k}^{ \pm} \widehat{\Theta}^{l, k}
\end{aligned}
$$

Соответствуюшие проекции плоских производных имеют вид $\partial_{\alpha}^{ \pm}, \mathcal{D}_{\alpha}^{ \pm}, \widehat{\mathcal{D}}^{ \pm, \pm}$.

В центральном базисе можно получить следуюшие представления $N=2$ ковариантных автодуальных производных:

$$
\nabla_{\alpha}^{ \pm}=\mathcal{D}_{\alpha}^{ \pm}, \quad \widehat{\nabla}^{+, \pm}=h^{-1} \widehat{\mathcal{D}}^{+, \pm} h, \quad h^{-1} \partial_{\alpha}^{+} h
$$

где $h(z, u)$ - киральный автодуальный мост, $\mathcal{D}_{\alpha}^{ \pm} h=0$.

Гармоническая связность для автодуального моста представляет собой киральноаналитический препотенциал этого формализма:

$$
h \mathcal{D}^{++} h^{-1}=v^{++}, \quad\left(\partial_{\alpha}^{+}, \mathcal{D}_{\alpha}^{ \pm}, \widehat{\mathcal{D}}^{+, \pm}\right) v^{++}=0
$$

Подчеркнем, что автодуальные решения обладают комбинированной аналитичностью. Автодуальный препотенциал можно интерпретировать как неограниченную матричную функцию координат $y^{\alpha+}, u_{i}^{ \pm}$и $\widehat{\Theta}^{+, \pm}$, которые параметризуют обшее $N=2$ автодуальное решение.

В калибровочной группе $S U(2)$ можно выбрать простую подстановку для автодуального препотенциала

$$
\left(v^{++}\right)_{i}^{k}=u^{+k} u_{i}^{+} b^{0}+\left(u^{+k} u_{i}^{-}+u^{-k} u_{i}^{+}\right) b^{++},
$$

где $b^{0}$ и $b^{++}$- вещественные кирально-аналитические функции. Мост-матрица для этой подстановки вычисляется через гармонические квадратуры [10]. В простом примере этой параметризации с $b^{0}=0$ и

$$
b^{++}=Y^{\alpha+} Y^{\beta+} \rho_{\alpha \beta}+\widehat{\Theta}^{+,-} Y^{\alpha+} u_{l}^{+} \lambda_{\alpha}^{l}
$$

решение зависит от постоянных тензорного и спинорного коэффициентов.

Нетрудно связать автодуальные представления для различных методов гармонизации суперполевых уравнений

$$
e^{-v} \widehat{\mathcal{D}}^{+,+} e^{v}=h^{-1} \widehat{\mathcal{D}}^{+,+} h, \quad \mathcal{D}_{\alpha}^{-}\left(e^{-v} \widehat{\mathcal{D}}^{+,+} e^{v}\right)=h^{-1} \partial_{\alpha}^{+} h
$$




\section{6. АВТОДУАЛЬНЫЕ СТАТИЧЕСКИЕ $N=2$ РЕШЕНИЯ}

Рассмотрим теперь дополнительное условие в BPS-уравнении (3.5) $r=p$, тогда эти уравнения могут быть преобразованы в простую связь:

$$
\begin{aligned}
-2 i A_{t}+W+\bar{W} & \equiv\left(\left(\bar{D}^{+} D^{+}\right)+\left(\bar{D}^{+}\right)^{2}-\left(D^{+}\right)^{2}\right) V^{--}= \\
& =-\frac{1}{2}\left(D^{\alpha+}-\bar{D}^{\alpha+}\right)\left(D_{\alpha}^{+}-\bar{D}_{\alpha}^{+}\right) V^{--}=0 .
\end{aligned}
$$

Можно также рассмотреть эквивалентные связи второго порядка на $V^{--}$, используя преобразование $D_{\alpha}^{+} \rightarrow e^{i \rho / 2} D_{\alpha}^{+}$.

Удобно ввести новые псевдовешественные спинорные координаты статического суперпространства

$$
\begin{gathered}
\Theta^{\alpha \pm} \equiv \frac{1}{\sqrt{2}}\left(\theta^{\alpha \pm}+\bar{\theta}^{\alpha \pm}\right), \quad \widehat{\Theta}^{\alpha \pm} \equiv \frac{1}{\sqrt{2}}\left(\theta^{\alpha \pm}-\bar{\theta}^{\alpha \pm}\right), \\
\left(\Theta^{\alpha \pm}\right)^{\dagger}=\Theta_{\alpha}^{ \pm}, \quad\left(\widehat{\Theta}^{\alpha \pm}\right)^{\dagger}=-\widehat{\Theta}_{\alpha}^{ \pm} .
\end{gathered}
$$

Соответствуюшие преобразованные спинорные производные

$$
\begin{array}{ll}
\mathcal{D}_{\alpha}^{ \pm} \equiv \frac{1}{\sqrt{2}}\left(D_{\alpha}^{ \pm}-\bar{D}_{\alpha}^{ \pm}\right), & \left(\mathcal{D}_{\alpha}^{ \pm}\right)^{\dagger}=-\mathcal{D}^{\alpha \pm}, \\
\widehat{\mathcal{D}}_{\alpha}^{ \pm}=-\frac{1}{\sqrt{2}}\left(D_{\alpha}^{ \pm}+\bar{D}_{\alpha}^{ \pm}\right), & \left(\widehat{\mathcal{D}}_{\alpha}^{ \pm}\right)^{\dagger}=\widehat{\mathcal{D}}^{\alpha \pm}
\end{array}
$$

имеют следуюшую алгебру:

$$
\begin{aligned}
& \left\{\mathcal{D}_{\alpha}^{+}, \widehat{\mathcal{D}}_{\beta}^{-}\right\}=-\left\{\widehat{\mathcal{D}}_{\alpha}^{+}, \mathcal{D}_{\beta}^{-}\right\}=-\partial_{\alpha \beta}, \\
& \left\{\mathcal{D}_{\alpha}^{+}, \mathcal{D}_{\beta}^{-}\right\}=\left\{\widehat{\mathcal{D}}_{\alpha}^{+}, \widehat{\mathcal{D}}_{\beta}^{-}\right\}=0 .
\end{aligned}
$$

Рассмотрим 3D ковариантное представление координат евклидова суперпространства (см. ниже (Б.1))

$$
y^{\alpha \dot{\beta}} \Rightarrow y^{\alpha \beta}+\varepsilon^{\alpha \beta} y_{4} .
$$

Отметим, что алгебра спинорных производных (6.4) возникает при размерной редукции $y_{4}=0 N=2 \operatorname{HSS}_{\mathrm{E}}$ (см. приложение Б). Ясно, что преобразование (6.2) связывает эквивалентные $3 \mathrm{D}$ подпространства гармонических суперпространств типа Минковского и Евклида. Таким образом, $(3,1)$-уравнение (6.1) эквивалентно 3D пределу евклидова $N=2$ уравнения автодуальности (4.4)

$$
\left(\mathcal{D}^{+}\right)^{2} V^{--}=0, \quad y_{4}=0 .
$$

Компонентные статические автодуальные решения могут быть получены в следуюшей калибровке:

$$
\begin{aligned}
\mathcal{V}^{++}= & \frac{1}{2} \widehat{\Theta}^{\alpha+} \widehat{\Theta}_{\alpha}^{+} c(y)+\frac{i}{2} \Theta^{\alpha+} \widehat{\Theta}_{\alpha}^{+} a_{t}(y)+i \Theta^{\alpha+} \widehat{\Theta}^{\beta+} a_{\alpha \beta}(y)- \\
& -\widehat{\Theta}^{\alpha+} \widehat{\Theta}_{\alpha}^{+} \Theta^{\beta+} u_{k}^{-} \Psi_{\beta}^{k}(y),
\end{aligned}
$$


где все поля эрмитовы, $\left(c, a_{t}, a_{\alpha \beta}, \Psi_{\alpha}^{k}\right)^{\dagger}=\left(c, a_{t}, a^{\alpha \beta}, \Psi_{k}^{\alpha}\right)$.

Можно построить компонентные $3 \mathrm{D}$ автодуальные уравнения

$$
\begin{gathered}
F_{\alpha \beta}=-\nabla_{\alpha \beta} a_{t}, \\
\nabla^{\alpha \beta} \nabla_{\alpha \beta} c=2\left[a_{t},\left[a_{t}, c\right]\right]-\left\{\Psi_{k}^{\alpha}, \Psi_{\alpha}^{k}\right\}, \\
\nabla_{\beta \gamma} \Psi^{\gamma k}+\frac{i}{2}\left[a_{t}, \Psi_{\beta}^{k}\right]=0,
\end{gathered}
$$

где

$$
\nabla_{\alpha \beta} c \equiv \partial_{\alpha \beta} c-i\left[a_{\alpha \beta}, c\right], \quad F_{\alpha \beta}=\partial_{\alpha \rho} a_{\beta}^{\rho}+\partial_{\beta \rho} a_{\alpha}^{\rho}-i\left[a_{\alpha \rho}, a_{\beta}^{\rho}\right] .
$$

Отметим, что статическая напряженность калибровочного поля и поле $a_{t}$ связаны автодуальным BPS-уравнением.

Суперполевой анализ статических автодуальных уравнений может быть проделан по аналогии с анализом евклидовых $4 \mathrm{D}$ автодуальных уравнений, приведенным в разделе 4 . Статический предел представления мост-матришы имеет вид

$$
A_{\alpha}^{ \pm}=0, \quad \hat{A}_{\alpha}^{+}(v)=e^{-v} \widehat{\mathcal{D}}_{\alpha}^{+} e^{v},
$$

где $v$ - статическая автодуальная мост-матрица, $\mathcal{D}_{\alpha}^{+} v=0$.

По аналогии с $4 \mathrm{D}$ автодуальным уравнением можно использовать формальную идентификацию всех групп $S U(2)$ в $3 \mathrm{D} \mathrm{HSS}_{\mathrm{E}} y^{\alpha \beta} \rightarrow y^{i k}, \Theta_{l}^{\alpha} \rightarrow \Theta_{l}^{k}, \widehat{\Theta}^{\alpha l} \rightarrow \widehat{\Theta}^{l, k}$. В этом случае препотенциал будет функцией одной гармонической проекции четной координаты.

ПРИЛОЖЕНИЕ А

\section{Гармоническое $N=2, D=(3,1)$ суперпространство}

$S U(2) / U(1)$-гармоники [1] параметризуют сферу $S^{2}$. Они образуют $S U(2)$-матрицу $u_{i}^{ \pm}$и определены по модулю $U(1)$. $S U(2)$-инвариантные гармонические производные действуют на гармониках

$$
\left[\partial^{++}, \partial^{--}\right]=\partial^{0}, \quad\left[\partial^{0}, \partial^{ \pm \pm}\right]= \pm 2 \partial^{ \pm \pm} .
$$

Специальное $S U(2)$-ковариантное сопряжение гармоник сохраняет $U(1)$-заряды:

$$
\widetilde{u_{i}^{ \pm}}=u^{ \pm i}, \quad \widetilde{u^{ \pm i}}=-u_{i}^{ \pm} .
$$

На гармонические производные произвольных гармонических функций $f(u)$ это сопряжение действует следуюшим образом:

$$
\widetilde{\partial^{ \pm \pm} f}=\partial^{ \pm \pm} \tilde{f}
$$

Рассмотрим координаты $N=2$ суперпространства $M(3,1 \mid 8)$ над $D=(3,1)$ пространством Минковского

$$
\begin{gathered}
z^{M}=\left(x^{\alpha \dot{\beta}}, \theta_{k}^{\alpha}, \bar{\theta}^{\dot{\alpha} k}\right), \\
\left(x^{\alpha \dot{\beta}}\right)^{\dagger}=x^{\beta \dot{\alpha}}, \quad\left(\theta_{k}^{\alpha}\right)^{\dagger}=\bar{\theta}^{\dot{\alpha} k},
\end{gathered}
$$


где $\alpha, \dot{\alpha}-S L(2, \mathbb{C})$-индексы. Можно определить аналитическое $\mathrm{HSS}_{\mathrm{M}}$ с двумя гармоническими размерностями $u_{i}^{I}$ и следуюшим множеством 4 четных и $(2+2)$ нечетных координат:

$$
\begin{aligned}
\zeta & =\left(x_{A}^{\alpha \dot{\beta}}, \theta^{\alpha+}, \bar{\theta}^{\dot{\alpha}+}\right), \\
x_{A}^{\alpha \dot{\beta}} & =x^{\alpha \dot{\beta}}-i\left(u_{k}^{-} u_{l}^{+}+u_{l}^{-} u_{k}^{+}\right) \theta^{\alpha k} \bar{\theta}^{\dot{\beta} l}, \\
\theta^{\alpha \pm} & =\theta^{\alpha k} u_{k}^{ \pm}, \quad \bar{\theta}^{\dot{\alpha} \pm}=\bar{\theta}^{\dot{\alpha} k} u_{k}^{ \pm} .
\end{aligned}
$$

Это суперпространство ковариантно по отношению к преобразованиям $N=2$ суперсимметрии

$$
\delta x_{A}^{\alpha \dot{\beta}}=2 i \theta^{\alpha+} \bar{\epsilon}^{\dot{\beta} k} u_{k}^{-}+2 i \bar{\theta}^{\dot{\beta}+} \epsilon^{\alpha k} u_{k}^{-}, \quad \delta \theta^{\alpha \pm}=\epsilon^{\alpha k} u_{k}^{ \pm}, \quad \delta \bar{\theta}^{\dot{\beta} \pm}=\bar{\epsilon}^{\dot{\beta} k} u_{k}^{ \pm} .
$$

Сопряжение нечетных аналитических координат имеет вид

$$
\theta^{\alpha \pm} \rightarrow \bar{\theta}^{\dot{\alpha} \pm}, \quad \bar{\theta}^{\dot{\alpha} \pm} \rightarrow-\theta^{\alpha \pm},
$$

а координаты $x_{A}^{\alpha \dot{\beta}}$ вещественны.

Рассмотрим простейшие спинорные и гармонические производные в этих координатаx:

$$
\begin{aligned}
D_{\alpha}^{+} & =\partial_{\alpha}^{+}, \quad \bar{D}_{\dot{\alpha}}^{+}=\bar{\partial}_{\dot{\alpha}}^{+}, \\
D^{++} & =\partial^{++}-i \theta^{\alpha+} \bar{\theta}^{\dot{\beta}+} \partial_{\alpha \dot{\beta}}+\theta^{\alpha+} \partial_{\alpha}^{+}+\bar{\theta}^{\dot{\alpha}+} \bar{\partial}_{\dot{\alpha}}^{+},
\end{aligned}
$$

где частные производные удовлетворяют соотношениям

$$
\partial_{\alpha \dot{\alpha}} x^{\beta \dot{\beta}}=2 \delta_{\alpha}^{\beta} \delta_{\dot{\alpha}}^{\dot{\beta}}, \quad \partial_{\alpha}^{+} \theta^{\beta-}=\delta_{\alpha}^{\beta}, \quad \bar{\partial}_{\dot{\alpha}}^{+} \bar{\theta}^{\dot{\beta}-}=\delta_{\dot{\alpha}}^{\dot{\beta}} .
$$

Можно также построить остальные гармонические и грассмановы производные в этих координатах:

$$
\begin{gathered}
D^{--}=\partial^{--}-i \theta^{\alpha-} \bar{\theta}^{\dot{\beta}-} \partial_{\alpha \dot{\beta}}+\theta^{\alpha-} \partial_{\alpha}^{-}+\bar{\theta}^{\dot{\alpha}-} \bar{\partial}_{\dot{\alpha}}^{-} \\
D_{\alpha}^{-}=-\partial_{\alpha}^{-}+i \bar{\theta}^{\dot{\beta}-} \partial_{\alpha \dot{\beta}}, \quad \bar{D}_{\dot{\alpha}}^{-}=-\bar{\partial}_{\dot{\alpha}}^{-}-i \theta^{\alpha-} \partial_{\alpha \dot{\alpha}}, \\
\partial_{\alpha}^{-} \theta^{\beta+}=\delta_{\alpha}^{\beta}, \quad \bar{\partial}_{\dot{\alpha}}^{-} \bar{\theta}^{\dot{\beta}+}=\delta_{\dot{\alpha}}^{\dot{\beta}}
\end{gathered}
$$

Полезно описать свойства этих производных по отношению к эрмитову сопряжению:

$$
\left(D^{++}, D^{--}\right) \rightarrow-\left(D^{++}, D^{--}\right), \quad D_{\alpha}^{ \pm} \rightarrow \bar{D}_{\dot{\alpha}}^{ \pm}, \quad \bar{D}_{\dot{\alpha}}^{ \pm} \rightarrow-D_{\alpha}^{ \pm} .
$$

Мы используем следующие соглашения:

$$
\begin{aligned}
\varepsilon^{i k} \varepsilon_{k l} & =\delta_{l}^{i}, & \varepsilon^{\alpha \gamma} \varepsilon_{\gamma \beta} & =\delta_{\beta}^{\alpha}, \\
\left(\theta^{ \pm}\right)^{2} & =\frac{1}{2} \theta^{\alpha \pm} \theta_{\alpha}^{ \pm}, & \left(\bar{\theta}^{ \pm}\right)^{2} & =\frac{1}{2} \bar{\theta}_{\dot{\alpha}}^{ \pm} \bar{\theta}^{\dot{\alpha} \pm}, \\
\left(D^{ \pm}\right)^{2} & =\frac{1}{2} D^{\alpha \pm} D_{\alpha}^{ \pm}, & \left(\bar{D}^{ \pm}\right)^{2} & =\frac{1}{2} \bar{D}_{\dot{\alpha}}^{ \pm} \bar{D}^{\dot{\alpha} \pm} .
\end{aligned}
$$


ПРИЛОЖЕНИЕ Б

\section{Гармоническое суперпространство в евклидовом случае}

Евклидово $N=2$ суперпространство $E(4 \mid 8)$ имеет координаты $z^{M}=\left(y^{\alpha \dot{\beta}}\right.$, $\left.\Theta_{k}^{\alpha}, \hat{\Theta}^{\dot{\alpha} k}\right)$ с альтернативными правилами сопряжения

$$
\left(y^{\alpha \dot{\alpha}}\right)^{\dagger}=y_{\alpha \dot{\alpha}}, \quad\left(\Theta_{k}^{\alpha}\right)^{\dagger}=\Theta_{\alpha}^{k}, \quad\left(\widehat{\Theta}^{\dot{\alpha} k}\right)^{\dagger}=\widehat{\Theta}_{\dot{\alpha} k},
$$

где $\alpha, \dot{\alpha}$ и $k$-индексы прямого произведения трех групп $S U(2)$. Евклидовы $N=2$ координаты аналитического суперпространства определены следуюшим образом:

$$
\begin{aligned}
\zeta_{\mathrm{E}} & =\left(y_{A}^{\alpha \dot{\beta}}, \Theta^{\alpha+}, \widehat{\Theta}^{\dot{\alpha}+}\right), \\
y_{A}^{\alpha \dot{\beta}} & =y^{\alpha \dot{\beta}}-\left(u^{k-} u_{l}^{+}+u_{l}^{-} u^{k+}\right) \Theta_{k}^{\alpha} \widehat{\Theta}^{\dot{\beta} l}, \\
\Theta^{\alpha \pm} & =\Theta_{k}^{\alpha} u^{k \pm}, \quad \widehat{\Theta}^{\dot{\alpha} \pm}=\widehat{\Theta}^{\dot{\alpha} k} u_{k}^{ \pm} .
\end{aligned}
$$

Евклидовы спинорные и гармонические производные в этих координатах имеют вид

$$
\begin{aligned}
\mathcal{D}_{\alpha}^{+} & =\frac{\partial}{\partial \Theta^{\alpha-}} \equiv d_{\alpha}^{+}, \quad \widehat{\mathcal{D}}_{\dot{\alpha}}^{+}=\frac{\partial}{\partial \widehat{\Theta}^{\dot{\alpha}-}} \equiv \hat{d}_{\dot{\alpha}}^{+}, \\
\mathcal{D}^{ \pm \pm} & =\partial^{ \pm \pm}-\Theta^{\alpha \pm} \widehat{\Theta}^{\dot{\beta} \pm} \partial_{\alpha \dot{\beta}}+\Theta^{\alpha \pm} d_{\alpha}^{ \pm}+\widehat{\Theta}^{\dot{\alpha} \pm} \hat{d}_{\dot{\alpha}}^{ \pm} \\
\mathcal{D}_{\alpha}^{-} & =-d_{\alpha}^{-}+\widehat{\Theta}^{\dot{\beta}-} \partial_{\alpha \dot{\beta}}, \quad \widehat{\mathcal{D}}_{\dot{\alpha}}^{-}=-\hat{d}_{\dot{\alpha}}^{-}-\Theta^{\alpha-} \partial_{\alpha \dot{\alpha}}
\end{aligned}
$$

Мы будем использовать следующие правила сопряжения для евклидовых гармонизованных нечетных координат и производных:

$$
\begin{array}{ll}
\left(\Theta^{\alpha \pm}\right)^{\dagger}=\Theta_{\alpha}^{ \pm}, & \left(\mathcal{D}_{\alpha}^{ \pm}\right)^{\dagger}=-\mathcal{D}^{\alpha \pm} \\
\left(\widehat{\Theta}^{\dot{\alpha} \pm}\right)^{\dagger}=-\widehat{\Theta}_{\dot{\alpha}}^{ \pm}, & \left(\widehat{\mathcal{D}}_{\dot{\alpha}}^{ \pm}\right)^{\dagger}=\widehat{\mathcal{D}}^{ \pm \dot{\alpha}} .
\end{array}
$$

Для билинейных комбинаций евклидовых спиноров справедливы следующие соглашения:

$$
\begin{array}{ll}
\left(\Theta^{ \pm}\right)^{2}=\frac{1}{2} \Theta^{\alpha \pm} \Theta_{\alpha}^{ \pm}, & \left(\widehat{\Theta}^{ \pm}\right)^{2}=\frac{1}{2} \widehat{\Theta}^{\dot{\alpha} \pm} \widehat{\Theta}_{\dot{\alpha}}^{ \pm}, \\
\left(\mathcal{D}^{ \pm}\right)^{2}=\frac{1}{2} \mathcal{D}^{\alpha \pm} \mathcal{D}_{\alpha}^{ \pm}, & \left(\widehat{\mathcal{D}}^{ \pm}\right)^{2}=\frac{1}{2} \widehat{\mathcal{D}}^{\dot{\alpha} \pm} \widehat{\mathcal{D}}_{\dot{\alpha}}^{ \pm} .
\end{array}
$$

Благодарности. Автор благодарен Е. Иванову и И. Нидерле за обсуждения. Эта работа частично поддержана грантами РФ ФИ № 99-02-18417, РФФИ-ННИО № 99-0204022, INTAS № 00254 и NATO PST.CLG № 974874, а также программой ВотрубаБлохинцев ЛТФ ОИЯИ. 


\section{Список литературы}

[1] A. Galperin, E. Ivanov, S. Kalitzin, V. Ogievetsky, E. Sokatchev. Class. Q. Grav. 1984. V. 1. P. 469.

[2] A. Galperin, E. Ivanov, V. Ogievetsky, E. Sokatchev. Class. Q. Grav. 1985. V. 2. P. 601.

[3] B. M. Zupnik. Phys. Lett. B. 1987. V. 183. P. 175.

[4] A. Galperin, E. Ivanov, V. Ogievetsky, E. Sokatchev. Ann. Phys. 1988. V. 185. P. 1.

[5] O. Ogievetsky. Harmonic representatives of instantons and self-dual monopoles. In: Group Theoretical Methods in Physics. Lect. Notes in Phys. V. 313. Eds. H.-D. Doebner, J.-D. Hennig, T. D. Palev. Berlin: Springer, 1988. P. 548

[6] C. Devchand, V. Ogievetsky. Nucl. Phys. B. 1994. V. 414. P. 763; Erratum. Nucl. Phys. B. 1995. V. 451. P. 768.

[7] B. M. Zupnik. Phys. Lett. B. 1996. V. 375. P. 170; Alternative formulations of $N=2$ supersymmetric gauge theory in harmonic superspace. In: Supersymmetry and Quantum Field Theory. Lect. Notes in Phys. V. 509. Eds. J. Wess, V. Akulov. Berlin: Springer, 1998. P. 157.

[8] B. M. Zupnik. Short harmonic superfields and light-cone gauge in super-Yang-Mills equations. In: Proc. Intern. Conf. "Quantization, Gauge Theory and Strings" (devoted to the memory of prof. E. S. Fradkin). Eds. A. Semikhatov, M. Vasiliev, V. Zaikin. M.: Scientific World, 2001. P. 277; hep-th/0011012.

[9] B. M. Zupnik. Nucl. Phys. B. 1999. V. 554. P. 365.

[10] B. M. Zupnik. Phys. Lett. B. 1988. V. 209. P. 513.

[11] N. Seiberg, E. Witten. Nucl. Phys. B. 1994. V. 426. P. 19.

[12] J. Gauntlett. Nucl. Phys. B. 1994. V. 411. P. 443.

[13] E. A. Ivanov, S. V. Ketov, B. M. Zupnik. Nucl. Phys. B. 1998. V. 509. P. 53.

[14] A. Semikhatov. Phys. Lett. B. 1983. V. 120. P. 171.

[15] I. V. Volovich. Phys. Lett. 1983. V. 129. P. 429; Lett. Math. Phys. 1983. V. 7. P. 517.

[16] Ю. И. Манин. Калибровочные поля и комплексная геометрия. М.: Наука, 1984.

[17] W. Siegel. Phys. Rev. D. 1995. V. 52. P. 1042.

[18] J. Niederle, B. Zupnik. Nucl. Phys. B. 2001. V. 598. P. 645; hep-th/0012114.

Поступила в редакцию 16.VII.2001 г. 